\title{
How to Prevent Type Flaw Attacks on Security Protocols
}

\author{
James Heather* Gavin Lowe ${ }^{\dagger} \quad$ Steve Schneider ${ }^{\ddagger}$
}

\begin{abstract}
A type flaw attack on a security protocol is an attack where a field that was originally intended to have one type is subsequently interpreted as having another type. A number of type flaw attacks have appeared in the academic literature. In this paper we prove that type flaw attacks can be prevented using a simple technique of tagging each field with some information indicating its intended type.
\end{abstract}

\section{Introduction}

A type flaw attack on a security protocol is an attack where a field that was originally intended to have one type is subsequently interpreted as having another type. For example, consider the seven-message version of the adapted Needham-Schroeder Public-Key Protocol [3]:

$$
\begin{array}{ll}
\text { Msg 1. } & a \rightarrow s: b \\
\text { Msg 2. } & s \rightarrow a:\{P K(b), b\}_{S K(s)} \\
\text { Msg 3. } & a \rightarrow b:\{n a, a\}_{P K(b)} \\
\text { Msg 4. } & b \rightarrow s: a \\
\text { Msg 5. } & s \rightarrow b:\{P K(a), a\}_{S K(s)} \\
\text { Msg 6. } & b \rightarrow a:\{n a, n b, b\}_{P K(a)} \\
\text { Msg 7. } & a \rightarrow b:\{n b\}_{P K(b)} .
\end{array}
$$

We adopt standard notation for protocols: $a$ and $b$ are agents' identities; $s$ is the identity of a trusted server; $n a$ and $n b$ are nonces; the functions $P K$ and $S K$ return an agent's

${ }^{*}$ Department of Computing, School of Electronics, Computing and Mathematics, University of Surrey, Guildford, Surrey GU2 7XH, UK; e-mail j . heather@eim.surrey.ac.uk.

${ }^{\dagger}$ Programming Research Group, Oxford University Computing Laboratory, Wolfson Building, Parks Road, Oxford OX1 3QD, UK; e-mail gavin. lowe@comlab.ox.ac.uk.

${ }^{\ddagger}$ Royal Holloway, University of London, Egham Hill, Egham, Surrey TW20 0EX, UK; e-mail steve@cs.rhul.ac.uk. 
public key and secret key, respectively; $\{m\}_{k}$ denotes $m$ encrypted by key $k$. We consider the above variables to be free variables that can be instantiated with different values in different runs.

Meadows, in [5], describes a type flaw attack on the unmodified NeedhamSchroeder Public-Key Protocol [6]. The adapted version of the protocol is vulnerable to essentially the same attack:

$$
\begin{aligned}
& \text { Msg } \alpha .3 . \quad I_{A} \rightarrow B:\left\{N_{I}, A\right\}_{P K(B)} \\
& \text { Msg } \alpha .4 . \quad B \rightarrow S: A \\
& \text { Msg } \alpha .5 . \quad S \rightarrow B:\{P K(A), A\}_{S K(S)} \\
& \text { Msg } \alpha .6 . \quad B \rightarrow I_{A}:\left\{N_{I}, N_{b}, B\right\}_{P K(A)} \\
& \operatorname{Msg} \beta .3 . \quad I_{\left(N_{b}, B\right)} \rightarrow A:\left\{N_{I},\left(N_{b}, B\right)\right\}_{P K(A)} \\
& \text { Msg } \beta .4 . \quad A \rightarrow I_{S}:\left(N_{b}, B\right) \\
& \text { Msg } \alpha .7 . \quad I_{A} \rightarrow B:\left\{N_{b}\right\}_{P K(B)} \text {. }
\end{aligned}
$$

The attack uses two runs, whose messages are labelled $\alpha$ and $\beta$; the notation $I_{A}$ denotes the penetrator $I$ either faking a message, apparently from $A$, or intercepting a message intended for $A$. The penetrator seeks to impersonate $A$ throughout run $\alpha$. When $B$ issues a nonce challenge at message $\alpha .6$, the penetrator replays this message at $A$, as message $\beta .3$. This is the first message $A$ receives, and so $A$ interprets it as the start of a new protocol run, taking the field $\left(N_{b}, B\right)$ to be an agent's identity, and so believes this message came from $\left(N_{b}, B\right)$. A therefore tries to request $\left(N_{b}, B\right)$ 's public key, by sending the "identity" $\left(N_{b}, B\right)$ to the server; this allows the penetrator to learn $N_{b}$, and hence respond to the nonce challenge.

In this paper, we consider a system where fields are tagged with some extra information indicating their intended type. One can think of the tag as a few bits attached to the field, with different bit patterns allocated to different types. For example, we will write "(nonce, $N)$ " to represent a value $N$ tagged in such a way to indicate that it intended as a nonce. We will similarly tag compound messages; for example, we will write "(pair, ((nonce, $N)$, (nonce, $\left.\left.N^{\prime}\right)\right)$ )" to represent a pair of values $N$ and $N^{\prime}$ tagged as nonces; we assume that the tag for a pair contains enough information to allow an honest agent to decompose the message correctly (for example, the tag might contain a representation of the number of bits in each component). We will assume that the tag for an encryption contains the type of the encrypting key and the type of the body of the encryption; for example, we will write "\{nonce, nonce $\}$ public" to represent a tag indicating an encryption of a pair of nonces with a public key.

We assume that honest agents will tag messages that they create with the type they intend them to have; for example, if they introduce a nonce, they will tag it as being a nonce. We assume that when an honest agent receives a message, it will check that all accessible tags (i.e. those tags not inside an encryption that this agent cannot decrypt) are as expected. On the other hand, we will not assume that the penetrator follows these tagging rules-we will allow the penetrator to place arbitrary tags upon 
accessible messages (i.e. those messages not protected by an encryption)—and we will not assume that honest agents can detect such dishonest tagging.

Existing implementations of certain protocols do include some basic tagging information, but this information is usually not systematically organised. In particular, the authors know of no current protocols that tag encryptions with the type of the body. As we shall show, without these encryption tags, the tagging system cannot guarantee to guard against type flaw attacks.

Suppose we were to use our tagging scheme in the attack described above. The text of message $\alpha .6$ would then become:

$$
\left(\{\mid \text { nonce, nonce, agent } \mid\}_{\text {public }},\left\{\left(\text { nonce, } N_{I}\right),\left(\text { nonce, } N_{b}\right),(\text { agent, } B)\right\}_{P K(A)}\right) \text {. }
$$

A penetrator could replace the outermost tag with

$$
\{\mid \text { nonce, agent } \mid\}_{\text {public }} \text {, }
$$

but the tags inside the encryption cannot be tampered with. Now $A$ will not accept this message as an instance of a message 3 , because in such messages the second field inside the encryption should be tagged as being an agent's identity; further, the body of the encryption should be tagged as containing three fields, not two. Hence this tagging scheme would prevent the above attack.

As another example, consider the Woo and Lam Protocol $\pi_{1}$ from [11]:

$$
\begin{array}{lll}
\text { Msg 1. } & a \rightarrow b: a \\
\text { Msg 2. } & b \rightarrow a: n b \\
\text { Msg 3. } & a \rightarrow b:\{a, b, n b\}_{\text {shared }(a, s)} \\
\text { Msg 4. } & b \rightarrow s:\left\{a, b,\{a, b, n b\}_{\text {shared }(a, s)}\right\}_{\text {shared }(b, s)} \\
\text { Msg 5. } & s \rightarrow b:\{a, b, n b\}_{\text {shared }(b, s)} .
\end{array}
$$

Here $\operatorname{shared}(a, s)$ denotes a key shared between $a$ and $s$. Note that $b$ cannot decrypt the message he receives in message 3 , but instead simply includes it inside message 4 . The following type flaw attack exploits this:

$$
\begin{array}{lc}
\text { Msg 1. } & I_{A} \rightarrow B: A \\
\text { Msg 2. } & B \rightarrow I_{A}: N_{b} \\
\text { Msg 3. } & I_{A} \rightarrow B: N_{b} \\
\text { Msg 4. } & B \rightarrow I_{S}:\left\{A, B, N_{b}\right\}_{\operatorname{shared}(B, S)} \\
\text { Msg 5. } & I_{S} \rightarrow B:\left\{A, B, N_{b}\right\}_{\text {shared }(B, S)} .
\end{array}
$$

The penetrator replays the nonce $N_{b}$ at $B$ in message 3, which $B$ accepts as being of the form

$$
\left\{A, B, N_{b}\right\}_{\text {shared }(A, S)}
$$


$B$ therefore encrypts $N_{b}$ within message 4 . However, this is precisely the form of message that the penetrator requires to fake message 5 .

If we were to again adopt the tagging scheme, the nonce in message 2 would become (nonce, $N_{b}$ ). The penetrator cannot replay the nonce in this form in message 3, but can retag the nonce with the tag that $B$ is expecting, to produce (\{|agent, agent, nonce $\mid\}_{\text {shared-key }}, N_{b}$ ). Message 4 would then become (dropping the "pair" tag and the pairing parentheses for ease of notation):

( $\left\{\text { agent, agent, }\{\text { agent, agent, nonce } \mid\}_{\text {shared-key }} \mid\right\}_{\text {shared-key }}$, $\left.\left\{(\text { agent, } A),(\text { agent, } B),\left(\{\text { agent, agent, nonce } \mid\}_{\text {shared-key }}, N_{b}\right)\right\}_{\text {shared }(B, S)}\right)$.

But this message could not now be replayed as an instance of message 5 , because $B$ is expecting a message where the third field inside the encryption is tagged as being a nonce. The penetrator could change the outermost tag, to create:

$$
\begin{aligned}
& (\{\text { agent, agent, nonce }\} \text { shared-key, } \\
& \left.\quad\left\{(\text { agent, } A),(\text { agent }, B),\left(\{\mid \text { agent, agent, nonce } \mid\}_{\text {shared-key }}, N_{b}\right)\right\}_{\text {shared }(B, S)}\right),
\end{aligned}
$$

but the penetrator cannot change the inner tag without access to the appropriate key. Again this tagging scheme prevents the attack. Observe that the type flaw attack is prevented simply by having the participants examine the tags; they do not need to be able to tell the true types of fields.

In this paper we prove that in fact this tagging scheme is enough to prevent all type flaw attacks. The utility of this result to the designers and implementers of protocols should be obvious.

This result is also useful to protocol analysers. Most protocol analysis techniques adopt the strong typing abstraction, where all messages considered in the analysis are assumed to be well-typed. This corresponds to an assumption that all agents can "magically" tell the true types of messages. The result of this paper presents a way of justifying this apparently unrealistic abstraction.

More precisely, we show the following:

If a protocol is secure under the strong typing abstraction, then it is secure under the tagging scheme.

In other words, this tagging scheme implements the strong typing abstraction. In fact, our approach is to show the following:

If there is an attack upon a protocol under the tagging scheme, then there is an attack under the tagging scheme such that all fields are correctly tagged.

(We make the concept of "correctly tagged" precise in the next section, but essentially it means that all fields are tagged with a tag that represents their true type.)

In the next section we describe how we can model protocols using tagged messages. Our model is based upon the strand space model of [10]. We use the strand 
space framework because it provides a particularly suitable notation for the kind of reasoning required for the proof. However, the results of the paper are general and can be applied to other approaches to the analysis of security protocols (in the context of the Dolev-Yao model) such as those in $[4,5,7,8]$.

In Section 3 we prove our claim that this tagging scheme prevents all type flaw attacks. We sum up, and discuss possible implementations for the tagging scheme, in Section 4.

\section{Modelling protocols}

In this section we present the model we will be using to prove the main result of this paper; this model will be based upon the strand space model of [10]. We describe how we model tagged facts ${ }^{1}$, and define what it means for a tagged fact to be correctly tagged. We then give a brief overview of the strand space model, showing how we model honest participants and penetrator capabilities. We also explain how breaches of security, and hence security properties, can be expressed in the strand space model.

\subsection{Tags and facts}

We assume some set Atom of atomic values, partitioned into types Agent, Nonce, PublicKey, etc. By partitioning the types in this way, we assume that each atomic value has a unique true type: for values introduced by an honest agent, this will be the type that the honest agent intended for the value; the penetrator, however, will be allowed to tag such values with a different type.

\section{Tags}

We assume that there is a tag corresponding to each base type; we will adopt obvious names for such tags. We will also assume tag "constructors" for pairing and encryption. The types of tags can be defined by:

$$
\operatorname{Tag}::=\text { agent } \mid \text { nonce } \mid \text { public }|\ldots| \text { pair | enc Tag* Tag. }
$$

We assume that the tag for an encryption includes an indication of the encryption algorithm (e.g. DES or RSA public key encryption) that is claimed to have been used to produce the message. We include this algorithm tag because we want to be able to model the case where a key is used in the wrong algorithm. More precisely, we associate algorithms with types of keys (e.g. associating RSA public key encryption with the type of RSA public keys) and include the tag for that key type within the encryption tag. We also include the type of the body (as a sequence of tags) within

\footnotetext{
${ }^{1}$ We use the term "fact" for messages or parts of messages, preferring to reserve the word "message" for complete messages of the protocol in question.
} 
the encryption tag, to enable agents to perform type checking on encrypted components that they cannot themselves decrypt. So enc $\left\langle t_{1}, \ldots, t_{n}\right\rangle k t$ will indicate a tag that claims that the accompanying fact is encrypted with a key of type $k t$ (using the appropriate algorithm), and where the body is a sequence of facts with tags $t_{1}, \ldots, t_{n}$; we will abbreviate this tag to $\left.\left\{\mid t_{1}, \ldots, t_{n}\right\}\right\}_{k t}$.

\section{Tagged facts}

We similarly define a type of tagged facts. We represent such tagged facts as (Tag, Fact) pairs, where the tag gives the claimed type of the fact; the facts themselves are built up from atoms using constructors for pairing and encryption.

$$
\begin{aligned}
\text { TaggedFact }::= & \text { Tag } \times \text { Fact } \\
\text { Fact }::= & \text { Atom } \mid \\
& \text { PAIR TaggedFact TaggedFact } \mid \\
& \text { ENCRYPT Tag TaggedFact Fact } .
\end{aligned}
$$

PAIR $t f 1$ tf 2 represents a fact formed by concatenating tagged facts $t f 1$ and $t f 2$. For example, a correctly tagged pair of nonces $(N 1, N 2)$ would be written as (pair, PAIR (nonce, $N 1)$ (nonce, $N 2)$ ). We will write $(t f 1, t f 2)$ as an abbreviation for (pair, PAIR $t f 1 t f 2$ ). We will drop superfluous parentheses within nested pairing that associates to the left, writing, for example, $(t f 1, t f 2, t f 3)$ for $((t f 1, t f 2), t f 3)$; we will drop such parentheses completely when the pair forms the body of an encryption.

ENCRYPT $k t$ tf $k$ represents tagged fact $t f$ encrypted using key $k$ and algorithm corresponding to $k t$; we will abbreviate this to $\{t f\}_{k}^{k t}$, and will tend to drop the $k t$ where it matches the type of $k$.

We adopt a version of the perfect encryption assumption [4], that is, that an honest agent can tell whether it has correctly decrypted a message, i.e. whether the decrypting key and algorithm it used to decrypt the message correspond to the encrypting key and algorithm used to create the message. This can be implemented by including sufficient redundancy within the encryption. We also assume that the cryptographic mechanisms exhibit no algebraic properties except those explicitly described in our model; for example, an encrypted message cannot be changed into another encrypted message by an agent who does not hold the decryption key (non-malleability).

Note that the type of the body of an encryption is included both inside and outside the encryption, for example:

$$
\left(\{\mid \text { agent, agent, nonce } \mid\}_{\text {shared-key }},\left\{(\text { agent }, A),(\text { agent, } B),\left(\text { nonce }, N_{b}\right)\right\}_{\text {shared }(B, S)}\right) \text {, }
$$

which is a shorthand for

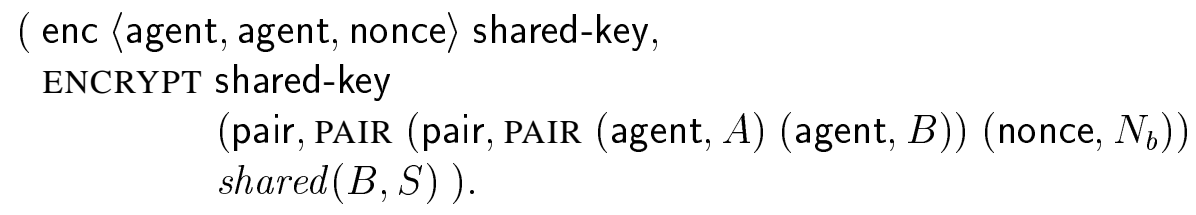


We will often want to talk about the tag or fact components from a tagged fact, so we define projection functions as follows:

$$
(t, f)_{1} \widehat{=} t, \quad(t, f)_{2} \widehat{=} f
$$

\section{Subfacts}

We will want to talk about the sub-tagged-facts of a tagged fact. The subfact relation is defined as the smallest relation such that:

- $t f \sqsubset t f$

- $t f \sqsubset(t,(t f 1, t f 2))$ if $t f \sqsubset t f 1$ or $t f \sqsubset t f 2$;

- $t f \sqsubset\left(t,\left\{t f^{\prime}\right\}_{k}\right)$ if $t f \sqsubset t f^{\prime}$.

We will also want to talk about the sub-untagged-facts of a tagged fact. We will write $f \sqsubset t f$ if $(t, f) \sqsubset t f$ for some tag $t$.

\section{Correct tagging}

We now define what it means for a tagged fact to be correctly tagged. We define this inductively over the structure of tags, as follows:

$$
\begin{aligned}
& \text { well-tagged(agent, } x) \Leftrightarrow x \in \text { Agent, } \\
& \text { well-tagged(nonce, } x) \Leftrightarrow x \in \text { Nonce, } \\
& \text { well-tagged(public, } x) \Leftrightarrow x \in \text { PublicKey, } \\
& \text { well-tagged(pair, } x) \Leftrightarrow \exists t f 1, t f 2: \text { TaggedFact • } \\
& x=\text { PAIR } t f 1 \text { tf } 2 \\
& \wedge \text { well-tagged } t f 1 \wedge \text { well-tagged } t f 2 \text {, } \\
& \text { well-tagged }\left(\{|t s|\}_{k t}, x\right) \Leftrightarrow \exists t f: \text { TaggedFact; } k: \text { Fact } \bullet \\
& x=\{t f\}_{k}^{k t} \wedge \text { well-tagged }(t f) \\
& \wedge \operatorname{well-tagged}(k t, k) \wedge t s=\text { get-tags } t f \text {, }
\end{aligned}
$$

where get-tags returns the sequence of tags labelling the body of an encryption:

$$
\begin{aligned}
\text { get-tags }(\text { pair, }(t f 1, t f 2)) & =\text { get-tags } t f 1 \frown \text { get-tags } t f 2, \\
\text { get-tags }(t, f) & =\langle t\rangle, \quad \text { for } t \neq \text { pair. }
\end{aligned}
$$

Note that for an encryption, the tag for the key must match the encryption algorithm used and also the type of the key used. 
It is also useful to characterize when a message is correctly tagged at the outermost level:

$$
\begin{aligned}
& \text { top-level-well-tagged(agent, } x) \Leftrightarrow x \in \text { Agent, } \\
& \text { top-level-well-tagged(nonce, } x) \Leftrightarrow x \in \text { Nonce, } \\
& \text {... } \\
& \text { top-level-well-tagged(pair, } x) \Leftrightarrow \exists t f 1, t f 2: \text { TaggedFact } \bullet x=\text { PAIR } t f 1 \text { tf } 2 \text {, } \\
& \text { top-level-well-tagged }\left(\{\mid t s\}_{k t}, x\right) \Leftrightarrow \exists t f: \text { TaggedFact } ; k: \text { Fact } \bullet \\
& x=\{t f\}_{k}^{k t} \wedge t s=\text { get-tags } t f .
\end{aligned}
$$

Note that for an encryption, the key tag must match the algorithm used for the encryption, but not necessarily the key used.

\subsection{Strand spaces}

As mentioned earlier, we will be using the strand space model from [10]. We give here a brief overview of the strand space model, and how we adapt it to deal with tagged facts.

A strand represents a sequence of communications by either an honest agent or the penetrator. Formally, it is a sequence of the form $\left\langle \pm t f_{1}, \pm t f_{2}, \ldots, \pm t f_{n}\right\rangle$, where $+t f$ represents the transmission of tagged fact $t f$ and $-t f$ represents reception of $t f$. A node is any particular communication $\pm t f$.

A graph structure is defined on strands by means of two types of edge:

- If nodes $n_{i}$ and $n_{i+1}$ are consecutive nodes on the same strand then we write $n_{i} \Rightarrow n_{i+1}$. This represents the chronological sequence of communications along a strand.

- If node $n_{i}=+t f$ and $n_{j}=-t f$ then we write $n_{i} \rightarrow n_{j}$. This captures communications from one strand to another.

A bundle represents a particular history of the network. Formally, if $\mathcal{C} \subseteq(\rightarrow \cup \Rightarrow)$ is a finite set of edges, and $\mathcal{N}_{\mathcal{C}}$ the set of nodes appearing on any edge in $\mathcal{C}$, then $\mathcal{C}$ is a bundle if:

1. whenever $n_{2} \in \mathcal{N}_{\mathcal{C}}$ and $n_{2}$ has negative sign, there exists a unique $n_{1}$ such that $n_{1} \rightarrow n_{2} \in \mathcal{C}$; note though that for each $n_{1}$ there may be any number (zero or more) of corresponding $n_{2}$ such that $n_{1} \rightarrow n_{2} \in \mathcal{C}$;

2. whenever $n_{2} \in \mathcal{N}_{\mathcal{C}}$ and $n_{1} \Rightarrow n_{2}$, we have $n_{1} \Rightarrow n_{2} \in \mathcal{C}$;

3. $\mathcal{C}$ is acyclic. 
Note that although the second condition requires that strands should start from the beginning - that is, that if some node of a strand is present in the bundle then so should the preceding nodes be present-it does not insist that the strand should run to completion.

We will want to be able to talk about when a fact or tagged fact is first transmitted. If $S$ is a set of tagged facts, then a node $n$ is an entry point to $S$ if the term of $n$ is $+t f$ for some $t f \in S$, and for each node $n^{\prime}$ previous to $n$ on the same strand, the term of $n^{\prime}$ is not in $S$. A tagged fact $t f$ will be said to originate on a node $n$ if $n$ is an entry point to the set $\left\{t f^{\prime} \mid t f \sqsubset t f^{\prime}\right\}$. Similarly, an untagged fact $f$ will be said to originate on a node $n$ if $n$ is an entry point to the set $\left\{t f^{\prime} \mid f \sqsubset t f^{\prime}\right\}$.

An untagged fact is uniquely originating in a bundle $\mathcal{C}$ if it originates on a unique node of $\mathcal{C}$. We will assume that all values of certain types are uniquely originating. We will call such types "fresh types"; examples will typically include nonces and session keys.

\subsection{Honest strands}

We assume that each role in the protocol is defined by a strand template: a sequence of templates for sent and received tagged facts, defining the operation of the agent in that role.

The templates for tagged facts will make use of some set Var of variables, and some set $F n$ of function identifiers (which will contain functions like $P K$, the public key function). Tagged fact templates can be defined as follows:

$$
\begin{aligned}
\text { TaggedTemplate }::= & \text { Tag } \times \text { Template } \\
\text { Template }:= & \text { Var | APPLY Fn Var* | } \\
& \text { PAIR TaggedTemplate TaggedTemplate | } \\
& \text { ENCRYPT Tag TaggedTemplate Template. }
\end{aligned}
$$

The template APPLY $g\left\langle v_{1}, \ldots, v_{n}\right\rangle$ represents the function $g$ applied to the variables $v_{1}, \ldots, v_{n}$; we will denote this $g\left(v_{1}, \ldots, v_{n}\right)$.

For example, the role played by $b$ in the Woo and Lam Protocol $\pi_{1}$ would be defined by the following sequence of templates (where we are adopting the same notational conventions as earlier):

$$
\begin{aligned}
& \text { temp } \widehat{=}\langle-(\text { agent }, a), \\
& + \text { (nonce, } n b) \text {, } \\
& -\left(\{\text { agent, agent, nonce }\}_{\text {shared-key }}, x\right) \text {, } \\
& +\left(\{\text { agent, agent, }\{\text { agent, agent, nonce }\}\}_{\text {shared-key }} \mid\right\}_{\text {shared-key }} \text {, } \\
& \left.\left\{(\text { agent, } a),(\text { agent }, b),\left(\{\text { agent, agent, nonce } \mid\}_{\text {shared-key }}, x\right)\right\}_{\text {shared }(b, s)}\right) \text {, } \\
& -(\{\text { agent, agent, nonce }\}\}_{\text {shared-key }} \\
& \left.\{(\text { agent, } a),(\text { agent }, b),(\text { nonce, } n b)\}_{\text {shared }(b, s)}\right) \\
& \rangle .
\end{aligned}
$$


Note that this strand uses five free variables: $a, b, s, n b$ and $x$; and also the function shared. Note also that the encrypted component of the third message, which $b$ does not decrypt, is represented by a variable $x$, modelling that $b$ should accept any value for this component.

All strands representing an execution of a particular role can be formed by instantiating the free variables of the corresponding template, that is, by substituting the free variables consistently with facts.

For example, in the case of the template above, a typical execution (where there has been no interference from the penetrator) can be formed using the substitution function sub where:

$$
\begin{gathered}
\operatorname{sub}(a)=A, \quad \operatorname{sub}(b)=B, \quad \operatorname{sub}(s)=S, \quad \operatorname{sub}(n b)=N_{b}, \\
\operatorname{sub}(x)=\left\{(\text { agent }, A),(\text { agent }, B),\left(\text { nonce }, N_{b}\right)\right\}_{\text {shared }(B, S)} .
\end{gathered}
$$

The execution in the attack described in Section 1 can be formed using the substitution function:

$$
\operatorname{sub}(a)=A, \quad \operatorname{sub}(b)=B, \quad \operatorname{sub}(s)=S, \quad \operatorname{sub}(n b)=N_{b}, \quad \operatorname{sub}(x)=N_{b} .
$$

(We argued above that it is not possible to produce penetrator strands to complete this attack.)

Implicit in the definition of a strand template is the assumption that when an honest agent first sees a free variable in a message that it receives, it will accept any value for the variable.

Formally, a substitution is a function:

$$
\text { sub }: \text { Var } \rightarrow \text { Fact. }
$$

Such a function can be lifted to complete tagged templates as follows ${ }^{2}$ :

$$
\begin{aligned}
\operatorname{sub}(t, v)= & (t, \operatorname{sub}(v)), \quad \text { for } v \in \operatorname{Var}, \\
\operatorname{sub}\left(t, g\left(v_{1}, \ldots, v_{n}\right)\right)= & \left(t, g\left(\operatorname{sub}\left(v_{1}\right), \ldots, \operatorname{sub}\left(v_{n}\right)\right)\right), \\
& \quad \text { for } g \in F n, \text { where the function application is defined, } \\
\operatorname{sub}(\text { pair, }(t f 1, t f 2))= & (\text { pair, }(\operatorname{sub}(t f 1), \operatorname{sub}(t f 2))), \\
\operatorname{sub}\left(\{|t s|\}_{t k},\{t f\}_{k}\right)= & \left.\{\mid t s\}_{t k},\{\operatorname{sub}(t f)\}_{\text {sub }(t k, k)_{2}}\right),
\end{aligned}
$$

and hence lifted to strand templates by applying $s u b$ to each message in turn.

We will assume that each strand template is consistently tagged, in the sense that the same tags are always given to the same variables. Formally, we define a type environment to be a function:

$$
\rho:(\operatorname{Var} \rightarrow T a g) \cup\left(F n \rightarrow \operatorname{Tag}^{*} \times T a g\right)
$$

The idea is:

\footnotetext{
${ }^{2}$ Recall that a subscript " 2 " extracts the fact component of a tagged fact.
} 
- for $v \in \operatorname{Var}, \rho(v)$ gives the tag that $\rho$ should receive, or, equivalently, the type with which $v$ should be instantiated;

- for $g \in F n, \rho(g)$ will be a pair of the form

$$
\left(\left\langle t_{1}, \ldots, t_{n}\right\rangle, t\right)
$$

where $t_{1}, \ldots, t_{n}$ give the types of the arguments of $f$, and $t$ gives the type of the result.

We can then define a tagged template to be well tagged with respect to $\rho$ as follows:

$$
\begin{aligned}
& \text { well-tagged }_{\rho}(t, v) \Leftrightarrow t=\rho(v), \quad \text { for } v \in \operatorname{Var}, \\
& \text { well-tagged }_{\rho}\left(t, g\left(v_{1}, \ldots, v_{n}\right)\right) \Leftrightarrow \rho(g)=\left(\left\langle\rho\left(v_{1}\right), \ldots, \rho\left(v_{n}\right)\right\rangle, t\right), \quad \text { for } g \in F n \text {, }
\end{aligned}
$$

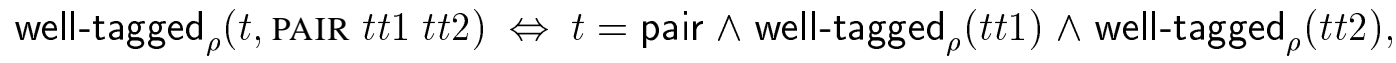

$$
\begin{aligned}
& \text { well-tagged } \left._{\rho}\left(t,\{t t\}_{k}^{k t}\right) \Leftrightarrow t=\{\mid \operatorname{get}-\operatorname{tags}(t t)\}\right\}_{k t} \wedge \operatorname{well}_{\text {-tagged }}(t t) \\
& \wedge \text { well-tagged }_{\rho}(k t, k) \text {, }
\end{aligned}
$$

where get-tags is defined analogously to earlier. Our assumption about strand templates being consistently tagged can be captured as follows:

Assumption 1. For each strand template temp, there is some type environment $\rho$ such that all the tagged fact templates of temp are well tagged with respect to $\rho$.

One corollary of this assumption is that each variable in a strand template receives a unique tag.

We will assume that the functions in $F n$ are partial, and defined only when they are applied to arguments of the correct types. For example, if we have a function $P K$ that is intended to return an agent's public key, $P K(N)$ will not be defined for a nonce $N$. We assume that if the arguments $v_{1}, \ldots, v_{n}$ of a function $g$ have tags $t_{1}, \ldots, t_{n}$, then $g\left(x_{1}, \ldots, x_{n}\right)$ will be defined only if $\left(t_{1}, x_{1}\right), \ldots,\left(t_{n}, x_{n}\right)$ are all well tagged.

We assume that the honest agents correctly follow the tagging scheme. This can be encapsulated in the following assumption:

Assumption 2. If the tagged fact $(t, f)$ originates on a regular strand, then top-levelwell-tagged $(t, f)$.

This assumption has a number of facets:

- If an agent introduces an atomic term for a variable, then it introduces a value of the expected type.

- An honest agent will tag a fact as being a pair only if it was indeed created as a pair. 
- An honest agent will tag a fact as being an encryption only if it is indeed created as an encryption; in this case the encryption tag will include the identity of the algorithm used, and the tags of the body; however, the key used for the encryption might not be of the expected type, because the honest agent might have received an ill-tagged key from the penetrator.

Recall that we designate certain types as fresh; honest agents should introduce new values for variables of fresh types. ${ }^{3}$

Assumption 3. If $v$ is a variable of a fresh type, which first appears in message $k$ of a strand template, then the value $s u b(v)$ with which it is instantiated does not instantiate any other variable appearing in any message up to and including message $k$; that is, $s u b(v)$ originates on this node.

Note that this assumption does not prevent the penetrator from replaying $s u b(v)$ in such a way that it instantiates a variable that first appears in a later message of the strand.

The above discussion has suggested that the bundle under consideration contains honest strands from a single protocol. In fact, this is not necessary: our results apply equally well when we consider bundles containing honest strands from several different protocols (as considered in [9]), provided that each protocol uses the same tagging scheme.

\subsection{Penetrator strands}

Following [10], we assume that there is some set $T$ of messages that the penetrator can produce himself. In [10] this is a set of atomic values; in contrast, we assume some larger set, including some compound facts; we will say more about this set below. We will also assume some set $K_{P}$ of keys that the penetrator has available.

Penetrator strands under the tagging scheme are exactly analogous to as in the standard strands model, but with the addition of a type of strand $\mathbf{R}$ representing manipulation of top-level tags. A penetrator strand is one of the following:

M Text message $\langle+(t, x)\rangle$ for $x \in T$, with $(t, x)$ well-tagged.

F Flushing $\langle-t f\rangle$.

T Tee $\langle-t f,+t f,+t f\rangle$.

C Concatenation $\left\langle-t f,-t f^{\prime},+\left(\right.\right.$ pair,$\left.\left.\left(t f, t f^{\prime}\right)\right)\right\rangle$.

S Separation $\left\langle-\left(\right.\right.$ pair, $\left.\left.\left(t f, t f^{\prime}\right)\right),+t f,+t f^{\prime}\right\rangle$

\footnotetext{
${ }^{3}$ This assumption complements the earlier definition of unique origination: it prevents, for example, the strand template $\langle-($ nonce, $n 1),+($ nonce, $n 2)\rangle$ being instantiated with $\langle-($ nonce, $N)$, $+($ nonce, $N)\rangle$.
} 
K Key $\langle+(t k, k)\rangle$ with well-tagged $(t k, k)$ and $k \in K_{P}$.

E Encryption $\left\langle-(t k, k),-t f,+\left(\{|t s|\}_{t k},\{t f\}_{k}^{t k}\right)\right\rangle$, where $t s=\operatorname{get}-\operatorname{tags}(t f)$.

D Decryption $\left\langle-\left(t k^{\prime}, k^{\prime}\right),-\left(\{t s\}_{t k},\{t f\}_{k}^{t k}\right),+t f\right\rangle$, where $t k$ and $t k^{\prime}$ are tags representing inverse key types, and $k^{\prime}$ is the decrypting key corresponding to $k$ when they are considered as keys of types $t k^{\prime}$ and $t k$, respectively.

R Retagging $\left\langle-(t, f),+\left(t^{\prime}, f\right)\right\rangle$.

Note that the retagging strand applies only to the top level tag, and that all other strands do not interfere with the tags of their messages. However, this does not necessarily prevent inner components from being retagged; for example, one component of a pair can be retagged by separation, retagging that component, and then concatenation.

Note that when the penetrator produces a fact, it is initially correctly tagged at the top level:

Lemma 1. If fact $f$ originates on a penetrator strand, then it does so with a tag $t$ such that top-level-well-tagged $(t, f)$.

Proof: The only strands that can be the origin of a fact are $\mathbf{M}, \mathbf{C}, \mathbf{K}$, and $\mathbf{E}$; in each case, the fact produced is indeed well-tagged at the top level.

Of course, the above does not prevent the penetrator from changing the tag after the fact is produced; the following lemma shows that the only place this retagging can occur is on a retagging strand.

Lemma 2. Every top-level-ill-tagged fact $(t, f)$ originates on a $\mathbf{R}$ strand.

Proof: Assumption 2 tells us that top-level-ill-tagged facts do not originate on honest strands. An argument similar to that in the previous lemma rules out all penetrator strands other than $\mathbf{R}$ strands.

\subsection{Security properties and attacks}

In this section we consider two typical security properties, namely secrecy and authentication, and produce generic definitions of each. Our definitions are obtained by generalizing those of Thayer Fábrega et al. [10], who consider these properties for specific protocols.

The definitions given by Thayer Fábrega et al. talk about properties that should hold of the protocol under the assumption that the penetrator does not have access to certain sets of keys. For example, the properties one would expect to hold will depend on whether or not an agent is running the protocol with the penetrator or someone whose secret key has been compromised. Our definition will generalize this to a set Keys, representing some keys that the penetrator may or may not have; this will be a set of function templates (templates of type APPLY $F n \operatorname{Var}^{*}$ ), for example 
$\{S K(a), S K(b)\}$, representing the secret keys of the agents $a$ and $b$. Given a substitution function $s u b$ for a particular strand, each key $k \in K$ eys will take the value $s u b(k)$. For example, if $\operatorname{sub}(a)=A$, then $\operatorname{sub}(S K(a))=S K(\operatorname{sub}(a))=S K(A)$. Recall that we assume that the penetrator has access to the set of keys $K_{P}$; hence we can say that the penetrator knows none of the keys as follows:

$$
\forall k \in K e y s \cdot \operatorname{sub}(k) \notin K_{P} .
$$

\section{Secrecy}

We now give a generic definition of secrecy. The definition will say that there is a breach of security when there is some strand (of some minimal length $h$ ) where certain keys have not been compromised, and the value of a particular variable $v$ (intended to remain secret) becomes known to the penetrator.

Definition 1. Let temp be the template for some role; let $(t, v)$ be a tagged variable of temp; let $h$ be a positive integer; and let Keys be a set of function templates. We define a failure of secrecy to be where each of the following holds:

1. There is a strand $s=\operatorname{sub}($ temp) with $\mathcal{C}$-height at least $h$ (i.e. at least the first $h$ messages of $s$ appear in the bundle $\mathcal{C}$ ).

2. $\forall k \in K e y s \cdot \operatorname{sub}(k) \notin K_{P}$.

3. There is a node in $\mathcal{C}$ with label $+\operatorname{sub}(t, v)$.

This definition is parameterized by temp, $v, h$ and Keys; in any given protocol, one would be interested in knowing whether this property holds for some particular values of these parameters.

\section{Authentication}

We now consider the property of authentication. We consider what it means for a particular role $r 2$ to be authenticated to another role $r 1$. For authentication to hold, we should expect that whenever there is a strand $s 1$ of $r 1$, there should be a "corresponding" strand $s 2$ of $r 2$; these strands should agree upon the identities of the agents involved, and possibly upon the values of some other variables (e.g. a session key that is established); we capture this aspect by specifying that the strands should agree on the values of all variables from some set $X^{4}$.

Definition 2. Let temp1 and temp2 be templates for two roles; let $X$ be a set of variables of those templates; let $h 1$ and $h 2$ be positive integers; and let Keys be a set of function templates. We define a failure of authentication to be where each of the following holds:

\footnotetext{
${ }^{4} \mathrm{We}$ are assuming that these variables are given the same names in the two different templates; this is not strictly necessary, but simplifies the notation.
} 
1. There is a strand $s 1=\operatorname{sub1}($ temp 1$)$ with $\mathcal{C}$-height at least $h 1$.

2. $\forall k \in K e y s \cdot \operatorname{sub1}(k) \notin K_{P}$.

3. There is no strand $s 2=s u b 2($ temp2) with $\mathcal{C}$-height at least $h 2$, such that $\forall x \in X \cdot \operatorname{sub1}(x)=\operatorname{sub2}(x)$.

This definition is parameterized by temp 1 , temp $2, X, h 1, h 2$ and Keys.

\section{How tagging prevents type flaw attacks}

In this section we prove our main result, that if there is an attack upon a protocol under the tagging scheme, then there is an attack under the tagging scheme such that all fields are correctly tagged. More precisely, we show that whenever there is an attack upon a protocol under the tagging scheme, then we can construct a renaming function $\phi$ over tagged facts, such that uniformly renaming all tagged facts under this function produces an attack in which all fields are correctly tagged.

Informally, if an honest agent is willing to accept some ill-tagged fact $(t, f)$, then that agent's behaviour must be essentially independent of $f$, and so he should be willing to accept any value in its place; in particular, he should be willing to accept the tagged fact $\phi(t, f)$ (which will have the tag $t$ ) in place of $(t, f)$. We proceed as follows:

1. In Section 3.1 we define the properties that $\phi$ must satisfy, and show that such a $\phi$ can always be constructed.

2. In Section 3.2 we show that if $S$ is an honest strand, then $\phi(S)$ (renaming all the facts of $S$ by $\phi$ ) is also an honest strand.

3. In Section 3.3 we similarly show that if $S$ is a penetrator strand, then there are one or two penetrator strands having the same set of tagged facts as $\phi(S)$, although possibly with a slightly different strand structure.

4. In Section 3.4 we show that given a bundle $\mathcal{C}$, there is a corresponding bundle $\mathcal{C}^{\prime \prime}$ where all terms are well-tagged; this bundle will contain the same set of terms on its nodes as $\phi(\mathcal{C})$, although possibly with a slightly different strand structure; we also show that facts from fresh types are uniquely originating in $\mathcal{C}^{\prime \prime}$ on the assumption that thay are uniquely originating in $\mathcal{C}$.

5. Finally, in Section 3.5 we show that if there is an attack in $\mathcal{C}$, then there is similarly an attack in $\mathcal{C}^{\prime \prime}$. 


\subsection{Defining the renaming function}

The following definition captures the required properties of $\phi$ :

Definition 3. Given a bundle $\mathcal{C}$, we define

$$
\phi: \text { TaggedFact } \rightarrow \text { TaggedFact }
$$

to be a renaming function for $\mathcal{C}$ if:

1. $\phi$ preserves top-level tags: if $\phi(t, f)=\left(t^{\prime}, f^{\prime}\right)$ then $t=t^{\prime}$.

2. $\phi$ returns well-tagged terms: well-tagged $(\phi(t f))$.

3. $\phi$ is the identity function over well-tagged terms: if well-tagged $(t f)$ then $\phi(t f)=t f$.

4. $\phi$ distributes through concatenations that are top-level-well-tagged:

$$
\phi(\text { pair, }(t f 1, t f 2))=(\text { pair, }(\phi(t f 1), \phi(t f 2))) .
$$

5. $\phi$ distributes through encryptions that are top-level-well-tagged:

$$
\phi\left(\{|t s|\}_{t k},\{t f\}_{k}^{t k}\right)=\left(\{\mid t s\}_{t k},\{\phi(t f)\}_{\phi(t k, k)_{2}}^{t k}\right), \quad \text { if } t s=\operatorname{get}-\operatorname{tags}(t f) .
$$

6. $\phi$ respects inverses of keys: if $k$ and $k^{\prime}$ are inverses of one another, when considered as keys of types $t k$ and $t k^{\prime}$, then $\phi(t k, k)$ and $\phi\left(t k^{\prime}, k^{\prime}\right)$ are also inverses of one another, when considered as keys of types $t k$ and $t k^{\prime}$.

7. If $(t, f)$ appears in $\mathcal{C}$, and $(t, f)$ is top-level-ill-tagged, then $\phi(t, f)_{2} \in T$. (Recall that $T$ is the set of messages the penetrator can produce himself.)

8. When $\phi$ is applied to a top-level-ill-tagged fact $t f$ of $\mathcal{C}$, it produces a fact that is essentially new, that is a fact that has no subfact in common with $\phi\left(t f^{\prime}\right)$ for any other fact $t f^{\prime}$ of $\mathcal{C}$ :

$$
\begin{aligned}
& \forall t f \in \operatorname{facts}(\mathcal{C}) \cdot \\
& \rightarrow \text { top-level-well-tagged }(t f) \wedge f \sqsubset \phi(t f) \Rightarrow \\
& \forall t f^{\prime} \in \operatorname{facts}(\mathcal{C}) \bullet t f \not \subset t f^{\prime} \Rightarrow f \not \subset \phi\left(t f^{\prime}\right),
\end{aligned}
$$

where $\operatorname{facts}(\mathcal{C})$ is the set of all the tagged facts and subfacts of nodes of $\mathcal{C}$.

Note that condition 8 implies that $\phi$ is injective over the facts of $\mathcal{C}$.

We now show that it is always possible to find such a $\phi$ :

Lemma 3. Given a bundle $\mathcal{C}$ there is some renaming function $\phi$ for $\mathcal{C}$. 
Proof: The following method gives a recipe for constructing such a $\phi$. We build the definition of $\phi$ from the bottom up, defining it over the subfacts of a fact before the fact itself. Consider, then, a tagged fact $(t, f)$, and suppose we have defined $\phi$ over all subfacts of $f$. We use a case analysis to construct $\phi(t, f)$ :

1. If $\neg$ top-level-well-tagged $(t, f)$ then pick a new value $f^{\prime}$ from $T$ (for condition 7 ) such that well-tagged $\left(t, f^{\prime}\right)$, and none of the atoms of $f^{\prime}$ has been used previously (for condition 8); define $\phi(t, f)=\left(t, f^{\prime}\right)$.

One proviso to this concerns keys: we should define $\phi$ over pairs of inverse keys simultaneously. If $k 1$ and $k 2$ are inverses of one another, when considered as keys of types $t k 1$ and $t k 2$, then pick $k 1^{\prime}$ and $k 2^{\prime}$ from $T$ such that: well-tagged $\left(t k 1, k 1^{\prime}\right)$ and well-tagged $\left(t k 2, k 2^{\prime}\right) ;\left(t k 1, k 1^{\prime}\right)$ and $\left(t k 2, k 2^{\prime}\right)$ are inverses; and none of the atoms of $k 1^{\prime}$ or $k 2^{\prime}$ has been used previously. Then define $\phi(t k 1, k 1)=\left(t k 1, k 1^{\prime}\right)$ and $\phi(t k 2, k 2)=\left(t k 2, k 2^{\prime}\right)$.

2. If well-tagged $(t, f)$ then define $\phi(t, f)=(t, f)$ (for condition 3 ).

3. If $(t, f)=($ pair, $(t f 1, t f 2))$ then define $\phi(t, f)=($ pair, $(\phi(t f 1), \phi(t f 2)))$ (for condition 4).

4. If $(t, f)=\left(\{t s\}_{t k},\{t f\}_{k}^{t k}\right)$ with $t s=$ get-tags $(t f)$ then define

$$
\phi(t, f)=\left(\{t s \mid\}_{t k},\{\phi(t f)\}_{\phi(t k, k)_{2}}^{t k}\right)
$$

(for condition 5).

The only assumption we need is that $T$ is big enough: we need to assume that the penetrator has access to sufficient supplies of values that he can always produce a new value in step 1. In practice, it is reasonable to assume that he is always able to produce new atomic facts, and use these to create new compound facts.

\subsection{Regular strands}

In this section we show that if $S$ is a regular strand, then so is $\phi(S)$. By definition, $S$ must be an instantiation of a strand template temp under a substitution function $s u b$. Consider the strand $S^{\prime}$ formed by instantiating temp using the substitution function $s u b^{\prime}$ defined by:

$$
s u b^{\prime}(v)=\phi(t, \operatorname{sub}(v))_{2} \quad \text { where } t \text { is the unique tag for } v \text { in temp. }
$$

Note that $S^{\prime}$ is also a regular strand, from our definition. The following lemma shows that the translation from $S$ to $S^{\prime}$ corresponds to a renaming under $\phi$, i.e. $\phi(S)=S^{\prime}$.

Lemma 4. Let temp, $\phi, s u b$ and $s u b^{\prime}$ be as above; then

$$
\phi(s u b(t e m p))=s u b^{\prime}(t e m p) .
$$


Proof: Let $t t$ be a tagged template in temp. We show that $\phi(s u b(t t))=s u b^{\prime}(t t)$. Assumption 1 allows us to proceed by induction over the structure of $t t$ as follows:

- Case $t t$ is a variable, say $t t=(t, v)$; then:

$$
\begin{aligned}
\phi(s u b(t t))= & \phi(t, \operatorname{sub}(v)) \\
= & \left\langle\text { by definition of } s u b^{\prime}\right\rangle \\
& \left(t, s u b^{\prime}(v)\right) \\
= & s u b^{\prime}(t t)
\end{aligned}
$$

- Case $t t$ is a function application, say

$$
t t=\left(t, g\left(v_{1}, \ldots, v_{n}\right)\right) .
$$

For $s u b(t t)$ to be defined, we must have that $\left(t_{1}, s u b\left(v_{1}\right)\right), \ldots,\left(t_{n}, s u b\left(v_{n}\right)\right)$ are all correctly tagged, where $t_{1}, \ldots, t_{n}$ are the tags for $v_{1}, \ldots, v_{n}$ in $t t$. Hence $\phi\left(t_{i}, \operatorname{sub}\left(v_{i}\right)\right)=\left(t_{i}, \operatorname{sub}\left(v_{i}\right)\right)$ for each $i$. So:

$$
\begin{aligned}
\phi(s u b(t t))= & \phi\left(t, g\left(\operatorname{sub}\left(v_{1}\right), \ldots, s u b\left(v_{n}\right)\right)\right) \\
= & \langle t t \text { is well tagged; using the above }\rangle \\
& \left(t, g\left(\phi\left(t_{1}, s u b\left(v_{1}\right)\right)_{2}, \ldots, \phi\left(t_{n}, \operatorname{sub}\left(v_{n}\right)\right)_{2}\right)\right) \\
= & \left\langle\text { definition of } s u b^{\prime}\right\rangle \\
& \left(t, g\left(s u b^{\prime}\left(v_{1}\right), \ldots, s u b^{\prime}\left(v_{n}\right)\right)\right) \\
= & s u b^{\prime}(t t) .
\end{aligned}
$$

- Case $t t$ is a pair, say $t t=($ pair, $(t f 1, t f 2))$; then:

$$
\begin{aligned}
\phi(s u b(t t))= & \phi(\text { pair, }(\operatorname{sub}(t f 1), \operatorname{sub}(t f 2))) \\
= & \langle\text { condition } 4 \text { of Definition } 3\rangle \\
& (\text { pair },(\phi(s u b(t f 1)), \phi(\operatorname{sub}(t f 2)))) \\
= & \langle\text { inductive hypothesis }\rangle \\
& \left(\text { pair },\left(s u b^{\prime}(t f 1), s u b^{\prime}(t f 2)\right)\right) \\
= & s u b^{\prime}(\text { pair },(t f 1, t f 2)) .
\end{aligned}
$$

- Case $t t$ is an encryption, say

$$
t t=\left(\left\{\left|t^{\prime}\right|\right\}_{k t},\left\{\left(t^{\prime}, f^{\prime}\right)\right\}_{k}\right) .
$$

Then:

$$
\begin{aligned}
\phi\left(\operatorname{sub}\left(\left\{\left|t^{\prime}\right|\right\}_{k t},\left\{\left(t^{\prime}, f^{\prime}\right)\right\}_{k}\right)\right)= & \phi\left(\left\{\left|t^{\prime}\right|\right\}_{k t}, \operatorname{sub}\left(\left\{\left(t^{\prime}, f^{\prime}\right)\right\}_{\text {sub }(k)}\right)\right. \\
= & \langle\text { condition 5 of Definition } 3\rangle \\
& \left(\left\{\left|t^{\prime}\right|\right\}_{k t},\left\{\phi\left(\operatorname{sub}\left(t^{\prime}, f^{\prime}\right)\right)\right\}_{\phi\left((k t, s u b(k))_{2}\right.}\right) \\
= & \langle\text { inductive hypothesis }\rangle \\
& \left(\left\{\left|t^{\prime}\right|\right\}_{k t},\left\{s u b^{\prime}\left(t^{\prime}, f^{\prime}\right)\right\}_{s u b^{\prime}(k t, k)_{2}}\right. \\
= & \operatorname{sub}^{\prime}\left(\left(\left\{\left|t^{\prime}\right|\right\}_{k t},\left\{\left(t^{\prime}, f^{\prime}\right)\right\}_{k}\right)\right) .
\end{aligned}
$$




\subsection{Penetrator strands}

We now show that given the penetrator strands of $\mathcal{C}$ and a renaming function $\phi$, we can construct corresponding strands in a bundle $\mathcal{C}^{\prime}$, formed by replacing each tagged fact $t f$ in $\mathcal{C}$ by $\phi(t f)$. We consider the possible cases one by one, giving the strand $S$ in $\mathcal{C}$ and its corresponding strand $S^{\prime}$ in $\mathcal{C}^{\prime}$. In the case of the $\mathbf{R}$ strand, we will change the strand structure, but we will keep the same set of (renamed) tagged facts.

M Text message Let $S=\langle+(t, x)\rangle$ with $x \in T$ and well-tagged $(t, x)$. Define $S^{\prime}=$ $\langle+\phi(t, x)\rangle$, which is an $\mathbf{M}$ strand because $\phi(t, x)=(t, x)$ and $x \in T$.

F Flushing Let $S=\langle-t f\rangle$. Define $S^{\prime}=\langle-\phi(t f)\rangle$, which is an $\mathbf{F}$ strand.

T Tee Let $S=\langle-t f,+t f,+t f\rangle$. Define $S^{\prime}=\langle-\phi(t f),+\phi(t f),+\phi(t f)\rangle$, which is a T strand.

\section{Concatenation}

Let $S=\langle-t f 1,-t f 2,+($ pair,$(t f 1, t f 2))\rangle$. Define

$$
S^{\prime}=\langle-\phi(t f 1),-\phi(t f 2),+\phi(\text { pair },(t f 1, t f 2))\rangle
$$

which is a valid concatenation strand, because

$$
\phi(\text { pair },(t f 1, t f 2))=(\text { pair },(\phi(t f 1), \phi(t f 2)))
$$

by condition 4 of Definition 3.

\section{S Separation}

Let $S=\langle-($ pair, $(t f 1, t f 2)),+t f 1,+t f 2\rangle$. Define

$$
S^{\prime}=\langle-\phi(\text { pair, }(t f 1, t f 2)),+\phi(t f 1),+\phi(t f 2)\rangle
$$

which is a valid separation strand, again by condition 4 of Definition 3 .

K Key Let $S=\langle+(t k, k)\rangle$ with well-tagged $(t k, k)$ and $k \in K_{P}$. Define $S^{\prime}=$ $\langle+\phi(t k, k)\rangle=\langle+(t k, k)\rangle$, which is a $\mathbf{K}$ strand.

\section{E Encryption}

Let $\left.S=\left\langle-(t k, k),-t f,+(\{t s\}\}_{t k},\{t f\}_{k}^{t k}\right)\right\rangle$ where $t s=$ get-tags $(t f)$. Define

$$
S^{\prime}=\left\langle-\phi(t k, k),-\phi(t f),+\phi\left(\{|t s|\}_{t k},\{t f\}_{k}^{t k}\right)\right\rangle
$$

which is a valid encryption strand because

$$
\phi\left(\{t s\}_{t k},\{t f\}_{k}^{t k}\right)=\left(\{|t s|\}_{t k},\{\phi(t f)\}_{\phi(t k, k)_{2}}^{t k}\right)
$$

by condition 5 of Definition 3. 


\section{Decryption}

Let $S=\left\langle-\left(t k^{\prime}, k^{\prime}\right),-\left(\{\mid t s\}_{t k},\{t f\}_{k}^{t k}\right),+t f\right\rangle$, with $k$ and $k^{\prime}$ inverses of one another. Define

$$
S^{\prime}=\left\langle-\phi\left(t k^{\prime}, k^{\prime}\right),-\phi\left(\{|t s|\}_{t k},\{t f\}_{k}^{t k}\right),+\phi(t f)\right\rangle
$$

which is a valid decryption strand, because

$$
\phi\left(\{t s \mid\}_{t k},\{t f\}_{k}^{t k}\right)=\left(\{|t s|\}_{t k},\{\phi(t f)\}_{\phi(t k, k))_{2}}^{t k}\right)
$$

by condition 5 of Definition 3 , and $\phi\left(t k^{\prime}, k^{\prime}\right)$ and $\phi(t k, k)$ are inverses of one another by condition 6 of Definition 3 .

R Retagging Let $S=\langle-(t 1, f),+(t 0, f)\rangle$. We proceed in two stages. We first construct the pair of strands $\langle-\phi(t 1, f)\rangle$, which is a strand of type $\mathbf{F}$, and $\langle+\phi(t 0, f)\rangle$. If $\neg$ top-level-well-tagged $(t 0, f)$ then this latter strand is of type $\mathbf{M}$, from condition 7 of Definition 3, and we are done; see Figure 1 for a depiction of the strands in $\mathcal{C}$ and $\mathcal{C}^{\prime}$.

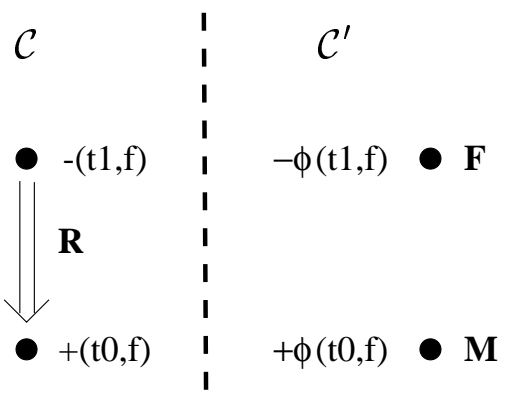

Figure 1: Replacing $\mathbf{R}$ strands (part 1)

Otherwise, top-level-well-tagged $(t 0, f)$, i.e. $S$ retags $f$ with a correct tag. Let $n 0$ and $n 0^{\prime}$ be the negative and positive nodes of $S$, respectively, as in the lefthand side of Figure 2. We show that some earlier $\mathbf{R}$ strand, possibly this one, has initial node labelled with $-(t 0, f)$. If $t 1=t 0$ we are done. Otherwise $(t 1, f)$ is top-level-ill-tagged, and so $(t 1, f)$ originated on another $\mathbf{R}$ strand, from Lemma 2. We proceed similarly with this $\mathbf{R}$ strand: let $n 1$ and $n 1^{\prime}$ be the two nodes, and let the term on the first node be $-(t 2, f)$; if $t 2=t 0$ we are done; otherwise $(t 2, f)$ originated on another $\mathbf{R}$ strand. Continuing in this way, we can form a sequence of earlier and earlier $\mathbf{R}$ strands, each retagging $f$. Because the bundle is finite, this process must eventually stop, by reaching an $\mathbf{R}$ strand where the first node, call it $n k$, has label $-(t 0, f)$. Let $n$ be the predecessor under $\longrightarrow$ of $n k$.

We can construct new strands in $\mathcal{C}^{\prime}$ as in the right-hand side of Figure 2. Nodes $n 0^{\prime}$ and $n k$ are removed; each of the other negative nodes on the $\mathbf{R}$ strands is 


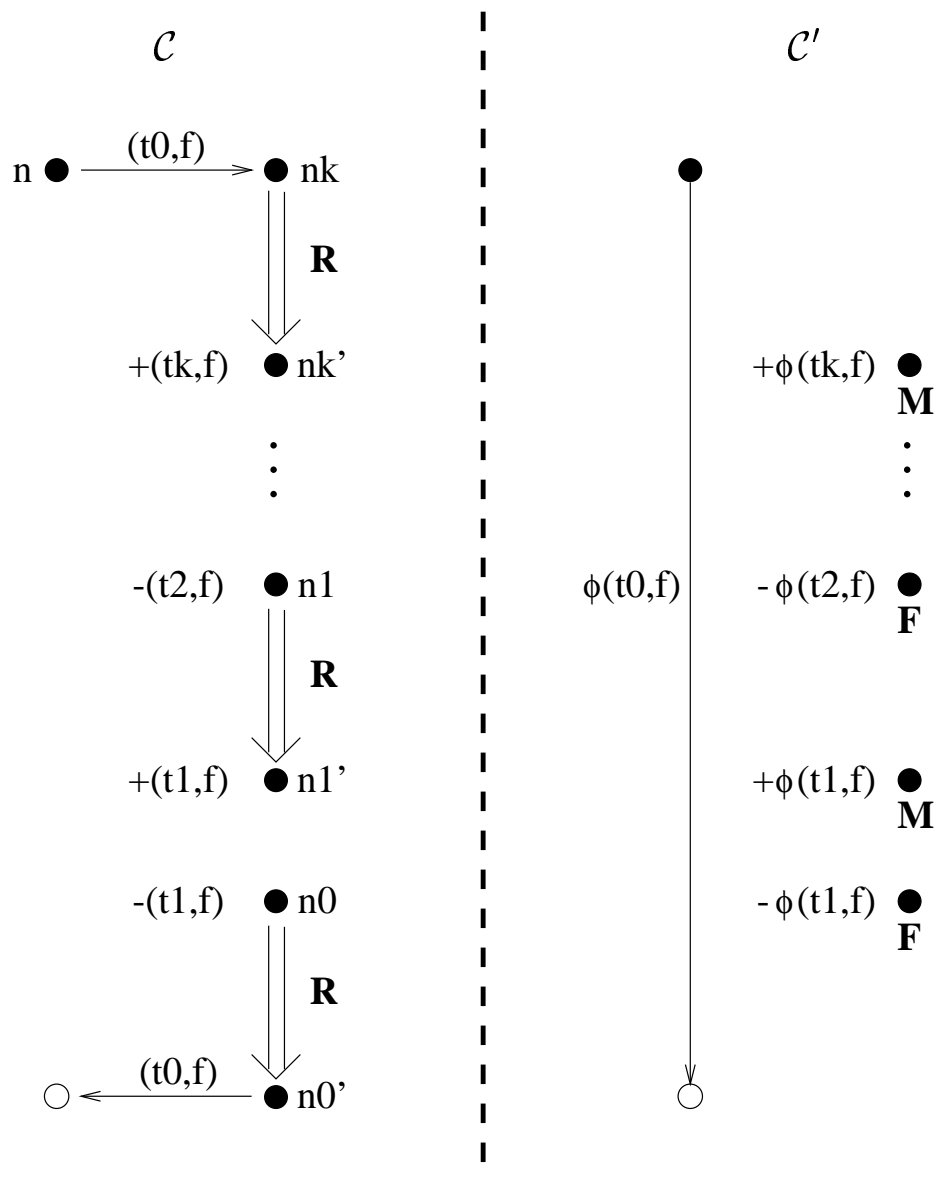

Figure 2: Replacing $\mathbf{R}$ strands (part 2) 
replaced by an $\mathbf{F}$ strand; each of the other positive nodes on the $\mathbf{R}$ strands is replaced by an $\mathbf{M}$ strand (these nodes were ill-tagged in $\mathcal{C}$, so the corresponding facts in $\mathcal{C}^{\prime}$ are elements of $T$ ); if $n 0^{\prime}$ had $\longrightarrow$ successors in $\mathcal{C}$ then the corresponding nodes in $\mathcal{C}^{\prime}$ becomes $\longrightarrow$ successors of $\phi(n)$.

\subsection{Bundles and unique origination}

We have described, above, how the nodes from bundle $\mathcal{C}$ are replaced with nodes in bundle $\mathcal{C}^{\prime}$. The graph structure of $\mathcal{C}^{\prime}$ is mostly the same as that of $\mathcal{C}$, with each edge of the form $+n \longrightarrow-n$ in $\mathcal{C}$ replaced by an edge $+\phi(n) \longrightarrow-\phi(n)$ in $\mathcal{C}^{\prime}$; the exception concerns $\mathbf{R}$ strands, which is dealt with above.

We now consider the question of unique origination. Recall that we assumed that all value of fresh types are uniquely originating in $\mathcal{C}$. We produce a bundle $\mathcal{C}^{\prime \prime}$ with the same honest strands as $\mathcal{C}^{\prime}$, but where all values of fresh types are again uniquely originating. In most cases, an origin of such a value in $\mathcal{C}^{\prime}$ corresponds to an origin in $\mathcal{C}$, and so in such cases the value is uniquely originating in $\mathcal{C}^{\prime}$; the exception concerns $\mathbf{R}$ strands, considered below.

Firstly, suppose $f_{0}$, of some fresh type, originates on an honest node in $\mathcal{C}^{\prime}$. Then it must do so instantiating some variable, $v$ say, that does not appear in any earlier message of the strand template. Suppose that $v$ is instantiated with $f_{1}$ on the corresponding node $n$ in $\mathcal{C}$. Then by Assumption 3, $f_{1}$ originates on $n$. Further, by Assumption 2, $f_{1}$ will be well tagged, and hence by condition 3 of Definition $3, f_{1}=f_{0}$. Thus if $f_{0}$ originates on an honest node in $\mathcal{C}^{\prime}$, then $f_{0}$ originates on the corresponding honest node in $\mathcal{C}$.

There are three circumstances under which $f_{0}$ can originate on a penetrator strand in $\mathcal{C}^{\prime}$ :

1. $f_{0}$ originates on an $\mathbf{M}$ strand corresponding to an occurrence of $f_{0}$ on the corresponding $\mathbf{M}$ strand in $\mathcal{C}$;

2. $f_{0}$ originates on a $\mathbf{K}$ strand corresponding to an occurrence of $f_{0}$ on the corresponding $\mathbf{K}$ strand in $\mathcal{C}$;

3. $f_{0}$ originates on an $\mathbf{M}$ strand corresponding to an occurrence of a top-level-illtagged term $(t, f)$ on an $\mathbf{R}$ strand in $\mathcal{C}$, with $f_{0} \sqsubset \phi(t, f)$.

The first two possibilities do not raise any problems, for if the term originates multiple times in $\mathcal{C}^{\prime}$ in these ways, then $f_{0}$ originates multiple times in $\mathcal{C}$. However, the third possibility introduces an origin not present in $\mathcal{C}$. Note that if one origin of $f_{0}$ corresponds to case 3 , then all origins of $f_{0}$ will correspond to case 3 , because of condition 8 of Definition 3. We produce a new bundle $\mathcal{C}^{\prime \prime}$ by merging those $\mathbf{M}$ strands, replacing them with a single such strand, whose successors under $\longrightarrow$ are the successors of the M strands in $\mathcal{C}^{\prime}$. 
Thus every origin of a value of a fresh type in $\mathcal{C}^{\prime \prime}$ corresponds to an origin in $\mathcal{C}$; such values originate uniquely in $\mathcal{C}$, and so they originate uniquely in $\mathcal{C}^{\prime \prime}$.

The results of the previous four subsections can be summarized in the following theorem:

Theorem 1. If $\mathcal{C}$ is a bundle (under our tagging scheme) then there is a renaming function $\phi$ and a bundle $\mathcal{C}^{\prime \prime}$, such that:

- $\mathcal{C}^{\prime \prime}$ contains the tagged facts of $\mathcal{C}$ (considered as a set), renamed by $\phi$;

- $\mathcal{C}^{\prime \prime}$ contains the same honest strands as $\mathcal{C}$, modulo the above renaming;

- values of fresh types are uniquely originating in $\mathcal{C}^{\prime \prime}$;

- all tagged facts in $\mathcal{C}^{\prime \prime}$ are well-tagged;

- $\mathcal{C}^{\prime \prime}$ contains no $\mathbf{R}$ strands.

\subsection{Attacks}

We now show that attacks upon the protocol are preserved by the transformation above. We show that if there is an attack in $\mathcal{C}$, then there is a corresponding attack in $\mathcal{C}^{\prime \prime}$; that is, essentially the same attack will work, but using well-tagged facts.

\section{Secrecy}

Following Definition 1, suppose there is an failure of secrecy in $\mathcal{C}$ as follows:

1. There is a strand $s=\operatorname{sub}($ temp $)$ with $\mathcal{C}$-height at least $h$.

2. $\forall k \in K e y s \cdot \operatorname{sub}(k) \notin K_{P}$.

3. There is a node $n$ with label $+s u b(t, v)$.

We show that there is a corresponding attack in $\mathcal{C}^{\prime \prime}$. Let substitution $s u b^{\prime}$ be defined as in Section 3.2:

$$
s u b^{\prime}(v)=\phi(t, s u b(v))_{2}, \quad \text { where } t \text { is the unique tag for } v \text { in temp. }
$$

Then:

1. There is a strand $s^{\prime}=s u b^{\prime}($ temp $)=\phi(\operatorname{sub}($ temp $))$ with $\mathcal{C}^{\prime \prime}$-height at least $h$, corresponding to $s$, from the way we have constructed the honest strands of $\mathcal{C}^{\prime \prime}$.

2. $\forall k \in$ Keys $\bullet s u b^{\prime}(k) \notin K_{P}$, because $s u b^{\prime}(k)=\operatorname{sub}(k)$ for such $k$.

3. The node corresponding to $n$ will have label $+s u b^{\prime}(t, v)=+\phi(s u b(t, v))$. 


\section{Authentication}

Following Definition 2, suppose there is an failure of authentication in $\mathcal{C}$ as follows:

1. There is a strand $s 1=\operatorname{sub1}($ temp 1$)$ with $\mathcal{C}$-height at least $h 1$.

2. $\forall k \in K e y s \cdot \operatorname{sub1}(k) \notin K_{P}$.

3. There is no strand $s 2=s u b 2($ temp2) with $\mathcal{C}$-height at least $h 2$, such that $\forall x \in X \cdot \operatorname{sub} 1(x)=\operatorname{sub2}(x)$.

We show that there is a corresponding attack in $\mathcal{C}^{\prime \prime}$. Let substitution $s u b 1^{\prime}$ be defined as in Section 3.2:

$s u b 1^{\prime}(v)=\phi(t, \operatorname{sub1}(v))_{2}, \quad$ where $t$ is the unique tag for $v$ in temp1.

Then:

1. There is a strand

$$
s 1^{\prime}=s u b 1^{\prime}(t e m p 1)=\phi(\operatorname{sub1}(\text { temp } 1))
$$

with $\mathcal{C}^{\prime \prime}$-height at least $h 1$, corresponding to $s 1$, from the way we have constructed the honest strands of $\mathcal{C}^{\prime \prime}$.

2. $\forall k \in$ Keys $\bullet \operatorname{sub1} 1^{\prime}(k) \notin K_{P}$, because $s u b 1^{\prime}(k)=\operatorname{sub1}(k)$ for such $k$.

3. There is no strand $s 2^{\prime}=s u b 2^{\prime}\left(\right.$ temp2) with $\mathcal{C}^{\prime \prime}$-height at least $h 2$ such that $\forall x \in X \bullet s u b 1^{\prime}(x)=\operatorname{sub2^{\prime }}(x)$. Suppose there were such an $s 2^{\prime}$; then, by the way we have constructed the honest strands in $\mathcal{C}^{\prime \prime}, s 2^{\prime}$ would correspond to some strand $s 2^{\prime \prime}=s u b 2^{\prime \prime}($ temp 2$)$ with $\mathcal{C}$-height at least $h 2$ such that:

$$
\begin{array}{r}
\forall v \in \operatorname{Var} \bullet s u b 2^{\prime}(v)=\phi\left(t, s u b 2^{\prime \prime}(v)\right)_{2} \\
\text { where } t \text { is the unique tag for } v \text { in temp } 2 .
\end{array}
$$

But then we would have for every $x \in X$ :

$$
\begin{aligned}
\phi(t, \operatorname{sub} 1(x))_{2} & =\operatorname{sub1}^{\prime}(x) \\
& =\operatorname{sub2}^{\prime}(x) \\
& =\phi\left(t, s u b 2^{\prime \prime}(x)\right)_{2}
\end{aligned}
$$

where $t$ is the tag for $x$ in temp 1 and temp2. But then by the injectivity of $\phi$ we would have $\operatorname{sub1}(x)=s u b 2^{\prime \prime}(x)$, contradicting part 3 of the assumption. 


\subsection{Example: the adapted Needham-Schroeder Public-Key Proto- col}

We now show how the results of this paper apply to the adapted Needham-Schroeder Public-Key Protocol, considered in Section 1. The two roles of this protocol can be defined by the strand templates:

$$
\begin{aligned}
& \text { Init } \widehat{=}\left\langle+\left(\{\text { nonce, agent } \mid\}_{\text {public-key }},\{(\text { nonce, } n a),(\text { agent }, a)\}_{P K(b)}\right),\right. \\
& -(\{\mid \text { nonce, nonce, agent }\} \text { public-key, } \\
& \left.\{(\text { nonce, } n a),(\text { nonce, } n b),(\text { agent }, a)\}_{P K(a)}\right) \text {, } \\
& \left.+\left(\{\mid \text { nonce } \mid\}_{\text {public-key }},\{(\text { nonce }, n b)\}_{P K(b)}\right)\right\rangle \text {, } \\
& \operatorname{Resp} \widehat{=}\left\langle-\left(\{\mid \text { nonce, agent } \mid\}_{\text {public-key }},\{(\text { nonce, } n a),(\text { agent }, a)\}_{P K(b)}\right)\right. \text {, } \\
& +\left(\{\mid \text { nonce, nonce, agent } \mid\}_{\text {public-key }}\right. \text {, } \\
& \left.\{(\text { nonce, } n a),(\text { nonce, } n b),(\text { agent }, a)\}_{P K(a)}\right) \text {, } \\
& \left.-\left(\{\mid \text { nonce } \mid\}_{\text {public-key }},\{(\text { nonce }, n b)\}_{P K(b)}\right)\right\rangle \text {. }
\end{aligned}
$$

The analysis of this protocol in [10] establishes a number of properties of the protocol, under the strong typing abstraction, and under the additional assumptions that the responder never introduces the same value for $n b$ as that received for $n a$, and that nonces are uniquely originating.

For example, Proposition 5.2 of that paper establishes, the following 'responder's guarantee': in any bundle in which there is a responder's strand $s 1=s u b 1($ Resp) such that $\operatorname{sub1}(S K(a)) \notin K_{P}$, there is a corresponding initiator's strand $s 2=s u b 2$ (Init) such that $s u b 1$ and $s u b 2$ agree on $a, b, n a$ and $n b$. This means that there is no failure of authentication under our Definition 2 (with temp $1=$ Resp, temp $2=$ Init, $X=\{a, b, n a, n b\}, h 1=3, h 2=3$ and Keys $=\{S K(a)\}$ in the notation of that definition).

We can immediately use the main result of this paper to deduce that the tagging scheme ensures that there is still no failure of authentication when the strong typing abstraction is dropped.

\section{Conclusions}

In this paper, we have shown that all type flaw attacks may be cheaply prevented by tagging each field with its intended type, and having honest participants check the tags of incoming messages. Our results generate no extra work in implementing protocols save adding a few extra bits of information into each message of the protocol; they generate no extra work in protocol analysis: protocols that have been proved correct under the strong typing abstraction are automatically secure under our scheme.

We have considered the properties of secrecy and authentication, but would expect the results to apply for other security properties, such as anonymity and nonrepudiation, that can also be expressed in terms of correspondences between agent strands. 
The key idea of the proof of the result was to show that any bundle corresponding to runs of the protocol can be transformed into another bundle in which all messages are correctly tagged, with equivalent strands of the protocol agents, and equivalent information available to the penetrator. Hence if there is such a bundle corresponding to an attack, then its transformation corresponds to an essentially similar but welltagged attack, demonstrating that the attack is not based around a type flaw. Such a transformation is possible because of the requirement that protocol agents check all tags they have access to. Ill-tagged messages will not affect the agent's behaviour in any essential way, and so might as well be replaced by messages that do have the correct tags. This is what is achieved by the renaming function $\phi$.

\subsection{Implementing the tagging scheme}

One possible approach to implementation would be to follow the structure of the Tag type. We could construct distinct bit patterns for each atomic type, and two more bit patterns to indicate a pair or encryption. Compound types could then be represented by concatenations of these basic bit patterns.

However, this would be somewhat inefficient, especially with complex types. A better approach is to identify all of the different types that are used in the execution of the protocol (or, in the case of a multi-protocol environment, all of the different types appearing in the execution of at least one protocol), and assign a unique tag number to each (with each tag number containing the same number of bits, to ensure unique readability).

Recall the seven-message version of the adapted Needham-Schroeder Public-Key Protocol:

$$
\begin{array}{ll}
\text { Msg 1. } & a \rightarrow s: b \\
\text { Msg 2. } & s \rightarrow a:\{P K(b), b\}_{S K(s)} \\
\text { Msg 3. } & a \rightarrow b:\{n a, a\}_{P K(b)} \\
\text { Msg 4. } & b \rightarrow s: a \\
\text { Msg 5. } & s \rightarrow b:\{P K(a), a\}_{S K(s)} \\
\text { Msg 6. } & b \rightarrow a:\{n a, n b, b\}_{P K(a)} \\
\text { Msg 7. } & a \rightarrow b:\{n b\}_{P K(b)} .
\end{array}
$$

This uses eight distinct types, and to each of these we could give a different tag number, representable in three bits:

$$
\begin{aligned}
\text { nonce } & =0, & \{\text { |public, agent } \mid\}_{\text {secret }} & =4, \\
\text { agent } & =1, & \{\mid \text { nonce, agent } \mid\}_{\text {public }} & =5, \\
\text { public } & =2, & \{\text { nonce, nonce, agent } \mid\}_{\text {public }} & =6, \\
\text { pair } & =3, & \{\text { nonce } \mid\}_{\text {public }} & =7 .
\end{aligned}
$$

These type identifiers would then appear as the initial part of each message, and inside pair types and encryption types. Under this approach, the implementation would 
become:

$$
\begin{array}{ll}
\text { 1. } & a \rightarrow s:(1, b) \\
\text { 2. } & s \rightarrow a:\left(4,\{(3,((2, P K(b)),(1, b)))\}_{S K(s)}\right) \\
\text { 3. } & a \rightarrow b:\left(5,\{(3,((0, n a),(1, a)))\}_{P K(b)}\right) \\
\text { 4. } & b \rightarrow s:(1, a) \\
5 . & s \rightarrow b:\left(4,\{(3,((2, P K(a)),(1, a)))\}_{S K(s)}\right) \\
6 . & b \rightarrow a:\left(6,\{(3,(0, n a),(3,((0, n b),(1, a))))\}_{P K(a)}\right) \\
7 . & a \rightarrow b:\left(7,\{(0, n b)\}_{P K(b)}\right) .
\end{array}
$$

As discussed earlier, we would still need to add information to the pair tag giving the lengths of the components, and we would need to add redundancy to the body of encryptions so as to implement the perfect encryption assumption.

It is important to realize that the results presented here do not depend for their validity on the exact type structure given in the paper.

The central theorem guarantees that using the tagging scheme will prevent attacks that rely on type confusion between two types that have distinct tags. Different tagging schemes distinguish different types, resulting in different guarantees about what type flaw attacks will be prevented. So, for instance, if we used a tagging scheme of

$$
\operatorname{Tag}::=\text { atom | pair | enc } \operatorname{Tag}^{*} \operatorname{Tag}
$$

then we would prevent all attacks involving passing off atoms as encryptions or pairs, but not attacks where a nonce is used in place of an agent's identity.

Furthermore, since the penetrator can always manipulate top-level tags and tags that are not protected by an encryption, such tags provide no useful guarantees and can be safely omitted. Thus in practice protocol implementations need tag only facts within encryptions, resulting in even less of a tagging overhead.

The tagging scheme could be simplified further, by combining the tags inside each encrypted component into a single component number. This is reasonably straightforward to prove using the technique of fault-preserving transformations from [2]: the protocol transformation from using component numbers to using tags is faultpreserving, which means that if a protocol of the former type is subject to an attack, then so is the protocol it maps to. Thus if the tagged version of the protocol has been proven correct, then the version using component numbers must be. In fact, using component numbers in this way provides more protection than simply tagging fields with their types, because it would prevent the penetrator replaying one component in the place of another with the same type, for example, replaying the encrypted component from message 3 of the Woo and Lam Protocol as a message 5. The advantages of not allowing one component to be replayed in the place of another are well understood; see, for example, Principle 10 of [1].

Note that including a message number within each encrypted component is not enough: different components within the same message require different component 
numbers. For example, consider the following protocol, which aims to authenticate $b$ to $a$ and to establish $n a$ as a secret shared between $a$ and $b$ :

$$
\begin{array}{ll}
\text { Msg 1. } & a \rightarrow b:\{a, c\}_{P K(b)},\{a, n a\}_{P K(b)} \\
\text { Msg 2. } & b \rightarrow a: c,\{n a\}_{P K(a)} .
\end{array}
$$

This protocol is correct under the strong typing assumption, and hence under the tagging scheme proposed in this paper. However, if each encrypted message is simply tagged with the message number in which it appears then the following attack exposes $N_{a}$ :

$$
\begin{array}{rrl}
\operatorname{Msg} \alpha .1 . & A \rightarrow I(B) & :\{1, A, C\}_{P K(B)},\left\{1, A, N_{a}\right\}_{P K(B)} \\
\operatorname{Msg} \alpha .1 . & I(A) \rightarrow B & :\{1, A, C\}_{P K(B)},\left\{1, A, N_{a}\right\}_{P K(B)} \\
\operatorname{Msg} \alpha .2 . & B \rightarrow A & : C,\left\{2, N_{a}\right\}_{P K(A)} \\
\operatorname{Msg} \beta .1 & I(A) \rightarrow B & :\left\{1, A, N_{a}\right\}_{P K(B)},\{1, A, C\}_{P K(B)} \\
\operatorname{Msg} \beta .2 & B \rightarrow I(A) & : N_{a},\{2, C\}_{P K(A)} .
\end{array}
$$

The attack exploits the fact that two parts of the same message have identical tags but different types. It may be prevented by replacing the message numbers with component numbers.

Protocol analysers using model checkers tend to assume that all messages are correctly typed, and so such techniques will not detect type flaw attacks. The results of this paper show how this can be overcome: the model checker tests for attacks that do not rely on type flaws, and the tagging scheme guarantees that the result can be extended to cover all type confusion attacks not considered by the model checking. In such situations, it is a simple matter to decide which tagging scheme to use in the implementation of the protocol: the tagging scheme should match the typing scheme in the model.

\section{Acknowledgements}

We would like to thank Joshua Guttman, Dave Cohen, and the anonymous referees for their useful comments on this paper.

This research was supported by grants from the UK Engineering and Physical Sciences Research Council, and the US Office of Naval Research. It was carried out while the first author was employed by Royal Holloway, University of London, and the second author by the University of Leicester.

\section{References}

[1] M. Abadi and R. Needham. Prudent engineering practice for cryptographic protocols. IEEE Transactions on Software Engineering, 22(1):6-15, 1996. Also in Proceedings of 
the 1994 IEEE Symposium on Security and Privacy, and Digital Equipment Corporation Systems Research Center, Research Report 125.

[2] M. L. Hui and G. Lowe. Fault-preserving simplifying transformations for security protocols. Journal of Computer Security, 9(1, 2):3-46, 2001.

[3] G. Lowe. Breaking and fixing the Needham-Schroeder public-key protocol using FDR. In Proceedings of TACAS, volume 1055 of Lecture Notes in Computer Science, pages 147-166. Springer Verlag, 1996. Also in Software-Concepts and Tools, 17:93-102, 1996.

[4] W. Marrero, E. Clarke, and S. Jha. A model checker for authentication protocols. In Proceedings of the DIMACS Workshop on Design and Formal Verification of Security Protocols, 1997. Available via URL: http://dimacs.rutgers.edu/Workshops / Security/program2/program.html.

[5] C. A. Meadows. Analyzing the Needham-Schroeder public-key protocol: A comparison of two approaches. In E. Bertino, H. Kurth, G. Martella, and E. Montolivo, editors, ESORICS '96, LNCS 1146, pages 351-364, 1996.

[6] R. Needham and M. Schroeder. Using encryption for authentication in large networks of computers. Communications of the ACM, 21(12):993-999, 1978.

[7] L. C. Paulson. The inductive approach to verifying cryptographic protocols. Journal of Computer Security, 6:85-128, 1998.

[8] S. A. Schneider. Verifying authentication protocols in CSP. IEEE Transactions on Software Enginnering, September 1998.

[9] F. J. Thayer Fábrega, J. C. Herzog, and J. D. Guttman. Mixed strand spaces. In 12th IEEE Computer Security Foundations Workshop, 1999.

[10] F. J. Thayer Fábrega, J. C. Herzog, and J. D. Guttman. Strand spaces: Proving security protocols correct. Journal of Computer Security, 7(2, 3):191-230, 1999.

[11] T. Y. C. Woo and S. S. Lam. A lesson on authentication protocol design. Operating Systems Review, 28(3):24-37, 1994. 